Józef Stala

ORCID: https://orcid.org/0000-0003-1776-6849

The Pontifical University of John Paul II in Krakow, Poland

\title{
Bericht über die internationale Wissenschaftskonferenz: "The Strong Family as the Foundation of a Healthy Society"
}

Vom 22. bis 23. Oktober 2018 fand in der Bibliothek der Päpstlichen Universität Johannes Paul II. in Krakau (UPJPII) die internationale Konferenz „The Strong Family as the Foundation of a Healthy Society” (Die starke Familie als Fundament einer gesunden Gesellschaft) statt. Als Organisatoren dieser Konferenz fungierten die Päpstliche Universität Johannes Paul II. in Krakau sowie die Polnische Gesellschaft für Familiologie.

Die Familie unterliegt in der heutigen Zeit gewaltigen Umgestaltungen - sowohl durch den Einfluss externer Faktoren als auch durch interne Veränderungen. Dieser Wandel betrifft ihre Struktur ebenso wie ihre Funktion. Gleichzeitig steht die Familie in enger Verbindung mit der Gesellschaft und erfüllt viele Aufgaben, die für die Entwicklung der Gesellschaft von Bedeutung sind. Denn in der Familie beginnt der Prozess der Weitergabe und der Aneignung von Kultur, Werten, grundlegenden Normen und Traditionen. Die Familie ist das System für modellhaftes soziales Verhalten, das Umfeld, in dem die familiären und außerfamiliären Rollen erlernt werden sowie die Schule sozialer Einstellungen. Eine starke Familie entscheidet über Gesundheit und Qualität der Gesellschaft. Eine richtig funktionierende Gesellschaft ist aufs engste mit dem Funktionieren einer starken, stabilen und glücklichen Familie verknüpft. Nur wenn die Familie gut funktioniert, können die grundlegenden Werte weiter bestehen, wodurch nicht nur die Kohärenz des Wertekanons sondern vor allem die sozialen Systeme erhalten bleiben. Im Hinblick auf die Rolle, welche die Familie im Leben 
des individuellen Menschen und in der gesamten Gesellschaft spielt, besteht der Bedarf nach wissenschaftlichen Reflexionen über ihre Realität. Diese betreffen einerseits die soziale Natur des Menschen, die sich am besten innerhalb der Familie verwirklichen kann, und andrerseits die Veränderung der gesellschaftlich-kulturellen Rahmenbedingungen ihrer Existenz und Funktionalität.

Die durchgeführte Konferenz sammelt und verbreitet die wissenschaftlichen Untersuchungen sowie das vorhandene Wissen aus dem Bereich der Familienwissenschaften. Ziele und angestrebte Effekte der Konferenz sind:

- ein geschärftes Bewusstsein dafür, was die Familie ausmacht und was ihre Rolle sowie Bedeutung für die Entwicklung der Gesellschaft sind;

- die Verbreitung des Wissens dazu, wie sehr die Qualität der Gesellschaft von einer starken Familie abhängt;

- den gegenseitigen Austausch wissenschaftlicher Forschungen, die im Rahmen der Disziplin Familienwissenschaften unternommen werden, um das familiäre Umfeld zu schützen und zu stärken;

- den Zugang zu den Untersuchungen und den vertieften Erkenntnissen zum Thema Ehe und Familie aus den Bereichen von Philosophie, Theologie, Psychologie, Pädagogik, Soziologie, Rechtswissenschaften sowie der Medizin ermöglichen;

- die Aufmerksamkeit auf das Wissen, das sich auf das Erkennen und das Gegensteuern bei Schwierigkeiten und Krisen in der Familie der Gegenwart bezieht;

- das Sammeln und die Weitergabe der Erkenntnisse darüber, welche Faktoren zum Aufbau einer starken und glücklichen Familie beitragen;

- die Suche nach neuen Formen der Arbeit und der Zusammenarbeit aller Institutionen und Lebensumfelder, die sich für die Familie einsetzen;

- die wissenschaftlichen Konsultationen und den Gedankenaustausch zwischen den Experten aus den Bereichen der Familienwissenschaften und der Sozialwissenschaften fördern.

Die Konferenz eröffnete H.H. Prof. Dr. habil. Józef Stala, Prorektor der UPJPII, der die Gäste begrüßte und in die Thematik einführte. Moderiert wurde die gesamte Konferenz, am ersten und am zweiten Tag, von Prof. Elżbieta Osewska, Präses der Polnischen Gesellschaft für Familiologie. Sie hielt auch das Eingangsreferat mit dem Titel: „What makes a family happy and strong?” (Was macht eine Familie glücklich und stark?). Die vier anschließenden Vorträge 
in englischer Sprache, die sich mit der Familientherapie für Opfer von Aggression oder innerfamiliärer Gewalt befassten, stellten folgende Wissenschaftler aus Slowenien (Universität Ljubljana) vor: Prof. Dr. Christian Gostečnik: "Violence in the marital relationship" (Gewalt in der ehelichen Beziehung); Prof. Dr. Barbara Simonič: "Emotional dynamic in the cycle of domestic violence" (Die emotionale Dynamik im Kreislauf der häuslichen Gewalt); Dr. Saša Poljak Lukek: "Why do I hit - the dynamics of physical punishment of children in the family" (Warum schlage ich - die Dynamik der physischen Bestrafung von Kindern in der Familie); Prof. Dr. Tanja Repič Slavič: "Effectiveness and efficiency of relational family therapy with victims of sexual violence" (Effektivität und Effizienz relationaler Familientherapie mit Opfern sexueller Gewalt). Danach griffen die Vertreter aus der Slowakei Fragen rund um die Schaffung einer Kultur von Ehe und Familie auf: Dr. Pavol Tománek (St. Elisabeth Universität für Gesundheit und Soziale Arbeit in Braislawa): "Family and current social, legal measures for its members in the Slovak Republic" (Familie und aktuelle soziale und gesetzliche Maßnahmen für ihre Mitglieder in der Republik Slowa$k e i)$; Dr. Richard Kucharčík (Katholische Universität Rosenberg): "Building a culture of marriages - anthropological and social arguments" (Der Aufbau einer Ehekultur - anthropologische und gesellschaftliche Argumente). Dagegen erörterte H.H. Dr. Mariusz Kuźniar (Tschechische Bischofskonferenz, Tschechische Republik) in seinem Referat „The relationship between the family and society in the Czech Republic" (Die Beziehung zwischen der Familie und der Gesellschaft in der Tschechischen Republik) die Frage nach den Beziehungen und den wechselseitigen Abhängigkeiten zwischen Familie und Gesellschaft am Beispiel Tschechiens. In den letzten beiden Vorträgen des ersten Tages gingen die Redner auf die Erziehungsfunktion der Familie ein: Prof. Dr. Herman Lombaerts (Katholische Universität Leuven, Belgien): "Home - School Cooperation" (Die Kooperation Elternhaus - Schule) sowie Dr. Nadia Delicata (Universität Malta): "The contemporary family in the digital culture" (Die Familie der Gegenwart in der digitalen Kultur).

Nach Abschluss der Konferenzpräsentationen fand die Hauptversammlung der Polnischen Gesellschaft für Familiologie statt, in deren Verlauf auch der erste Tag der Konferenz zusammengefasst wurde.

Der zweite Tag der Konferenz wurde vor allem durch Präsentationen von jungen Wissenschaftlern, Doktoranden aus dem Fachbereich Familienwissenschaften der Päpstlichen Universität Johannes Paul II. in Krakau und der Universität Oppeln gestaltet, ergänzt von Referaten mehrerer Dozenten beider 
Hochschulen: H.H. Prof. Paweł Landwójtowicz (Universität Oppeln): „Praca $\mathrm{z}$ traumą relacyjną w terapii par" (Arbeit am Beziehungstrauma in der Paartherapie); Dr. Urszula Miernik (Universität Oppeln): „Małoletnie macierzyństwo w kontekście polityki rodzinnej" (Die Mutterschaft Minderjähriger im Kontext der Familienpolitik); Anna Paneth (Doktorandin, UPJPII): „Działanie fundacji Rychwałd na rzecz rodziny" (Das Wirken der Stiftung Rychwałd in Sachen der Familie); Dr. Katarzyna Kutek-Sładek (UPJPII): „Funkcjonowanie społeczno-zawodowe dorosłych osób z niepełnosprawnościami i ich rodzin w Polsce" (Das gesellschaftlich-berufliche Funktionieren Erwachsener mit Behinderungen und ihrer Familien in Polen); Szymon Peterman (Doktorand, Universität Oppeln): „Antyspołeczne zachowania dzieci i młodzieży z rodzin dotkniętych przemocą domową" (Asoziale (Antigesellschaftliche) Verhaltensweisen von Kindern und Jugendlichen aus Familien, in denen es häusliche Gewalt gibt); Piotr Guzdek(Doktorand, Universität Oppeln): „Rodzina wobec pogrzebu dziecka zmarłego w poronieniu klinicznym" (Die Familie angesichts der Beerdigung eines Kindes, das nach einer klinischen Fehlgeburt verstorben ist); H.H. Prof. J. Stala (UPJPII): „Rodzina jak środowisko wychowania do wartości” (Die Familie als Umfeld für die Werteerziehung); Marzena Zięba (Doktorandin, UPJPII): „Zastosowanie zasady podwójnego skutku w rozeznawaniu decyzji o rozwodzie" (Die Anwendung des Grundsatzes beidseitiger Auswirkungen bei Aussagen zum Entschluss für die Scheidung).

Die Konferenz präsentierte die Familie als wichtiges Bindeglied zwischen dem privaten und dem öffentlichen Menschen, als Kreislauf der Generationen, aber vor allem unterstrich sie die wechselseitige Abhängigkeit zwischen einer starken, glücklichen, erfolgreich erziehenden Familie und einer stabilen, dauerhaften und starken Gesellschaft. Es wurde folgendes betont: Wenn es uns um die Stabilität und die Entwicklung der Gesellschaft, des Volkes und der Kirche geht, dann müssen wir die Familie der Gegenwart in allen Bereichen stärken. 